\title{
Reductive Defluorination of Graphite Monofluoride by Weak, Non-Nucleophilic Reductants Reveals Low-Lying Electron- Accepting Sites
}

\begin{tabular}{|r|l|}
\hline Journal: & Physical Chemistry Chemical Physics \\
\hline Manuscript ID & CP-ART-01-2018-000384.R1 \\
\hline Article Type: & Communication \\
\hline Date Submitted by the Author: & $17-$ Apr-2018 \\
\hline Complete List of Authors: & $\begin{array}{l}\text { Liu, Yijun; Tianjin Institute of Power Sources, National Key Laboratory of } \\
\text { Science and Technology on Power Sources; Indiana University } \\
\text { Bloomington, Department of Chemistry } \\
\text { Noffke, Benjamin; Indiana University Bloomington, Department of } \\
\text { Chemistry } \\
\text { Gao, Xinfeng; Indiana University, Chemistry } \\
\text { Losovyj, Yaroslav; Indiana University Bloomington, Department of } \\
\text { Chemistry; University of Nebraska-Lincoln, Department of Physics and } \\
\text { Astronomy } \\
\text { Cui, Yi; Indiana University-Purdue University Indianapolis, Department of } \\
\text { Mechanical Engineering } \\
\text { Fu, Yongzhi; Indiana University-Purdue University Indianapolis, } \\
\text { Department of Mechanical Engineering } \\
\text { Raghavachari, Krishnan; Indiana University, Chemistry } \\
\text { Siedle, Allen; Indiana University Bloomington, Department of Chemistry } \\
\text { Li, Liang-shi; Indiana University Bloomington, Department of Chemistry }\end{array}$ \\
\hline \hline
\end{tabular}




\section{Journal Name}

\section{COMMUNICATION}

\section{Reductive Defluorination of Graphite Monofluoride by Weak, Non-Nucleophilic Reductants Reveals Low-Lying Electron- Accepting Sites}

Received 00th January 20xx, Accepted 00th January 20xx

DOI: $10.1039 / x 0 x \times 00000 x$

\author{
Yijun Liu, ${ }^{a}$ Benjamin W. Noffke, ${ }^{a}$ Xinfeng Gao, ${ }^{a}$ Yaroslav Lozovyj, ${ }^{a}$ Yi Cui, ${ }^{b, c}$ Yongzhu Fu, ${ }^{b}$ Krishnan
} Raghavachari, ${ }^{a}$ Allen R. Siedle, ${ }^{\mathrm{a}, *}$ Liang-shi $\mathrm{Li}^{\mathrm{a}, *}$

www.rsc.org/

Graphite monofluoride (GF) can undergo reductive defluorination in the presence of weak, non-nucleophilic reductants. This leads to a new approach to GF-polyaniline composites as cathode materials for significantly improved discharge capacity of primary lithium batteries. We postulate that the reduction is mediated by residual $\pi$-bonds in GF.

Conversion between $\mathrm{sp}^{2}$ - and $\mathrm{sp}^{3}$-hybridized carbon is a common theme in modifying electrical, optical, chemical, or dispersion properties of graphitic carbon materials. ${ }^{1}$ Among graphite-derived $\mathrm{sp}^{3}$-carbon compounds, graphite monofluoride (GF, stoichiometry $\mathrm{CF}_{1}$ ), with a fluorine atom covalently linked to each carbon atom, is of particular interest. ${ }^{2} \mathrm{GF}$ can be reduced and defluorinated at room temperature by lithium to recover $\mathrm{sp}^{2}$-carbon, along with formation of LiF. ${ }^{3}$ Because of its large thermodynamic driving force, this reaction has been commercially applied to make lithium primary batteries with the highest energy-density among all lithium-based batteries. ${ }^{3-4}$ In addition, the reductive defluorination of GF and graphene fluoride, the fluorinated counterpart of graphene,$^{5}$ have been recently applied as precursors to graphene nanostructures with controlled dimensions and bandgap. ${ }^{6}$ Therefore, an improved understanding of the reductive defluorination of GF can lead to novel processes to control the reactions and may have practical significance.

Because of its structural complexity, understanding of the properties of GF are largely based on theoretical calculations with certain assumptions applied regarding its structure. ${ }^{7}$ For example, GF is generally considered as stacks of two-dimensional arrays of edgesharing cyclohexyl rings with a fluorine atom linked to each carbon atom. DFT calculations on such structures have shown that GF has the top of the valence band at approximately $7.7 \mathrm{eV}$ below vacuum and a valence-conduction bandgap approximately $7.4 \mathrm{eV}$, almost independent of the conformation of the cyclohexyl rings (i.e., chair

a. Department of Chemistry, Indiana University, Bloomington, Indiana, USA 47405.

${ }^{b}$. Department of Mechanical Engineering, Indiana University-Purdue University Indianapolis, Indianapolis, Indiana, USA 46202.

c. School of Mechanical Engineering, Purdue University, West Lafayette, Indiana, USA 47907.

+ Electronic Supplementary Information (ESI) available: experimental details, supplementary results, and theoretical calculations. See DOI: 10.1039/x0xx00000x or boat, etc.). ${ }^{8}$ This suggests that GF has a very low electron affinity close to $0.3 \mathrm{eV}$, and explains the needs for strong reductants such as alkaline metals for reductive defluorination of GF, unless the reductants are simultaneously capable of nucleophilic attack (e.g., hydrazine and iodide)..$^{4-5,9}$ Here we report new findings on reductive defluorination of GF by weak, non-nucleophilic, reductants. The observations have been applied to make a novel GF-polyaniline composite, leading to improved discharge capacity in primary lithium batteries. In addition we suggest that residual $\pi$-bonds in GF are the electron-accepting sites for triggering the defluorination reaction.

As mentioned above, we recently found, unexpectedly, that GF can be reduced by weak reductants with negligible nucleophilicity, indicating its high oxidizing power and existence of low-lying electron-accepting states (vide infra). This is illustrated by the color change when $N, N, N^{\prime}, N^{\prime}$-tetramethyl-p-phenylenediamine (TMPD, known as Wurster's blue), a weak non-nucleophilic reductant, was added to a GF suspension in acetonitrile at room temperature. Within a few seconds, the liquid phase turned blue, a characteristic color of radical cations of TMPD (i.e., TMPD ${ }^{*}$ ), suggesting oxidation of TMPD and reduction of GF (Figure 1a). This was subsequently confirmed by electron paramagnetic resonance spectroscopy (EPR) measurements at $298 \mathrm{~K}$ that revealed a hyperfine splitting pattern (Figure $1 \mathrm{~b}$ ) identical to that reported for the TMPD radical cations. ${ }^{10}$

GF can be reduced by even weaker reductants, though at decreased reaction rates. TMPD has a standard potential of $E^{0}=$ $+0.41 \mathrm{~V}$ vs. the standard hydrogen electrode $(\mathrm{SHE})^{11}$ in acetonitrile (or $4.85 \mathrm{~V}$ vs. the vacuum level${ }^{12}$ ). GF can oxidize other weak reductants, such as decamethylferrocene $\left(\mathrm{FeCp}_{2}{ }^{*}, E^{0}=+0.12 \mathrm{~V}\right.$ vs. $\mathrm{SHE}){ }^{13}$ tetrathiafulvalene (TTF, $E^{0}=+0.56 \mathrm{~V}$ vs. SHE), ${ }^{14}$ and decamethylosmocene $\left(\mathrm{OsCp}_{2}{ }^{*}, E^{0}=+0.64 \mathrm{~V}\right.$ vs. SHE). ${ }^{15}$ Under the same reaction conditions as for TMPD described above, for $\mathrm{FeCp}_{2}{ }^{*}$ the oxidation product can be observed within seconds with UVabsorption spectra (ESI, Figure S1), but for TTF it takes minutes for $\mathrm{TTF}^{\circ+}$ to be observed with EPR (Figure 1c). OsCp ${ }_{2}{ }^{*}$, an even weaker reductant, was barely able to reduce GF even days after mixing (ESI, Figure S1). In Figure $1 \mathrm{~d}$ we summarize the standard redox potentials of the reductants studied, together with the position of the bottom of the calculated conduction band of defect-free GF for comparison. ${ }^{8}$ Since there is a large discrepancy in the electron affinity of GF and the reduction potentials of the reductants, the observed reduction can only be explained by other acceptor states in GF (vide infra). 

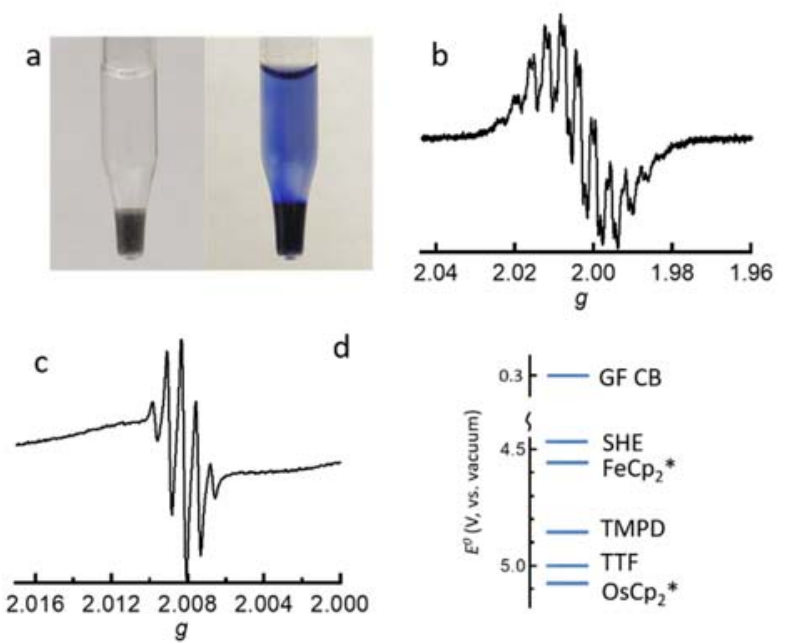

Figure 1. Reduction of GF by weak reductants. a, Color before (left) and after (right) TMPD is added to GF. The liquid is acetonitrile. b, EPR spectrum of TMPD'+ at $298 \mathrm{~K}$ as a result of oxidation of TMPD by GF. c, EPR spectrum of TTF*+ at $205 \mathrm{~K}$ as a result of oxidation of TTF by GF. d, Standard reduction potentials of the reductants in acetonitrile vs. the vacuum level. For comparison, position of the bottom of the calculated conduction band (CB) of GF is also shown. ${ }^{8}$

Meanwhile, reduction of GF by the weak reductants releases fluoride to the liquid phase of the reaction mixtures. For example, Figure $2 a$ shows the fluorine-NMR spectrum of the supernatant of the reaction mixtures with TMPD, revealing appearance of soluble fluorine-containing species after the reaction. The chemical shifts of the peaks ( -130 and $-152 \mathrm{ppm}$, relative to $\left.\mathrm{CFCl}_{3}\right)$ correspond to $\mathrm{SiF}_{6}{ }^{2-}$ and $\mathrm{BF}_{4}{ }^{-}$, respectively, due to corrosion of the borosilicate glass NMR tube by $\mathrm{F}^{-}$. The same results were also observed in reaction mixtures with $\mathrm{FeCp}_{2}{ }^{*}$ or TTF (ESI, Figure S2). Further, the solids from the reaction mixture showed decreased fluorine content and formation of $\mathrm{sp}^{2}$-hybridized carbon. X-ray photoelectron spectroscopy (XPS) measurements of the solid product obtained with TMPD showed $\mathrm{F} / \mathrm{C}=0.71$ after a week, significantly lower than the starting $\mathrm{F} / \mathrm{C}$ atomic ratio of 1.0 (ESI, Table S1). The Raman spectrum of this material ( $514.5 \mathrm{~nm}$ excitation, Figure $2 \mathrm{~b}$ ) showed new peaks at 1330 (FWHM 50) and 1600 (FWHM 30) $\mathrm{cm}^{-1}$ (intensity ratio 1.1:1). This can be interpreted as either graphene with an extremely high defect density ${ }^{16}$ or a continuous network of $\mathrm{C}=\mathrm{C}$ bonds, indicating conversion of $\mathrm{sp}^{3}$-carbon in $\mathrm{GF}$ to $\mathrm{sp}^{2}$-carbon. This is consistent with the color change of the solids from the original pale gray to black, as can be visualized in Figure 1a. However, X-ray diffraction measurements did not reveal any long-range structural order or graphite formation.

The surprising observation of GF reduction by weak reductants prompted us to apply GF as an oxidant for synthetic purposes. In particular, we applied it to make a GF-polyaniline composite material with increased electrical conductivity for the Li/CF battery. Graphite fluoride with various fluorine content has important applications as cathode materials for primary batteries. ${ }^{3 a}, 4$ In particular, the lithium/graphite fluoride (denoted $\mathrm{Li} / \mathrm{CF}_{x}, 0<x<1.5$ ) primary battery employs lithium as the anode, and, because of the light mass and small radii of lithium and fluoride ions, has one of the highest theoretical specific energies (e.g., $2180 \mathrm{Wh} / \mathrm{kg}$ for $x=1.0$ ) among solid cathode systems. In addition, the Li/CF battery has very slow self- discharge ( $0.5 \%$ per year) and very long shelf life ( $>10$ years), making it desirable for devices that require long battery life such as in cardiac pacemakers as well as some military, aerospace, and electronic devices. ${ }^{4} \mathrm{~A}$ challenge that limits the application of the $\mathrm{Li} / \mathrm{CF}_{\mathrm{x}}$ battery has been the poor electrical conductivity of the $\mathrm{CF}_{\mathrm{x}}$ cathode. ${ }^{4}$ This leads to a number of drawbacks such as low output power and heat generation during discharging, etc. a

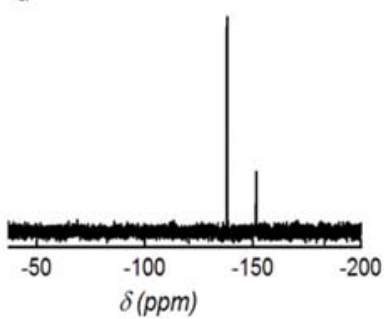

b

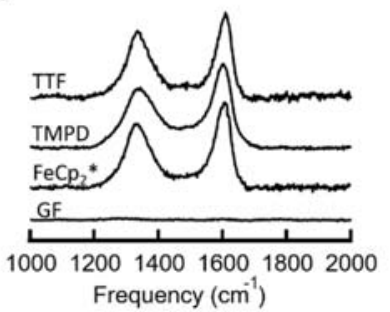

Figure 2. Defluorination of GF by weak reductants. a, An F-NMR spectrum of the supernatant from the GF-TMPD reaction mixture. b, Raman spectra ( $514.5 \mathrm{~nm}$ excitation) of the solid products after GF was exposed to various reductants compared with that of the starting GF.

The GF-polyaniline composite material was synthesized by dispersing GF (as received, stoichiometry $\mathrm{CF}_{1.1}$ ) in a solution of aniline in an acidified acetonitrile/water mixture, followed by stirring in air for 30 days at room temperature. Similar to reactions described above, the GF powders turned black, with the supernatant turning violet. The violet color is characteristic of pernigraniline, a highly oxidized form of polyaniline. ${ }^{17}$ The solids in the reaction mixtures were subsequently washed repetitively to remove unreacted aniline and soluble reaction products until the supernatant appeared colorless. IR spectra of the solid products in $\mathrm{KBr}$ pellets confirmed the presence of polyaniline in the solid products of the GF-aniline reaction (ESI, Figure S5). Further, XPS showed that the C/F atomic ratio changed from the original $1 / 1.1$ to $1 / 0.80$ (ESI, Figure S4). From the XPS-measured $\mathrm{N}$ content, we estimated the weight percentage of polyaniline in the solids to be less than $4 \%$. Therefore, mixing aniline with GF produces $\mathrm{sp}^{2}$-carbon from partial reduction of the GF as well as conjugated polyaniline due to the oxidative polymerization of aniline, both of which could contribute to electrical conductivity of the composite material. Because electron transfer occurs over a short distance, we speculate that the polymerization occurs only at the solid/liquid interface, leading to intimate mixing of polyaniline with partially reduced GF. In contrast, in a control experiment, aniline stirred under the same reaction conditions without GF led to a brown solution, characteristic of air-oxidized aniline. ${ }^{18}$

We subsequently made Li/CFx coin cells with either GF or the GF-polyaniline composite as the cathode materials, and tested their discharge characteristics. For the cathode, either of the two materials were mixed with carbon black, with polyvinylidene fluoride (PVDF) binder at the weight ratio of 85:10:5. Lithium metal sheet was used as the anode, and coin cells were assembled under identical conditions (details in ESI). Shown in figure $3 a$ are the Nyquist plots of the impedance of the cells measured between $200 \mathrm{kHz}$ to $0.1 \mathrm{~Hz}$ (black for GF and red for GF-polyaniline). As indicated by the intercepts on the $x$-axis (i.e., real part of the impedance, $Z^{\prime}$ ), the cells made with the GF-polyaniline composite have internal resistance only a third of that of cells made with GF (6.2 $\Omega$ vs. $17.9 \Omega$ ). We attribute it to contributions from both the $\mathrm{sp}^{2}$-carbon formed in reduced GF and the polyaniline in the composite, having intimate 
electrical contact. This leads to superior discharge capacity, as shown in Figure 3b. At the discharge rate of $C / 50$, cells made with GFpolyaniline yielded a specific capacity of $659 \mathrm{mAh} \mathrm{g}^{-1}$ (for voltage drop from the initial $2.5 \mathrm{~V}$ to $1.5 \mathrm{~V}$ ). This is significantly higher than $526 \mathrm{mAh} \mathrm{g}^{-1}$ for cells made with GF, despite the lower fluorine content in the GF-polyaniline composite.
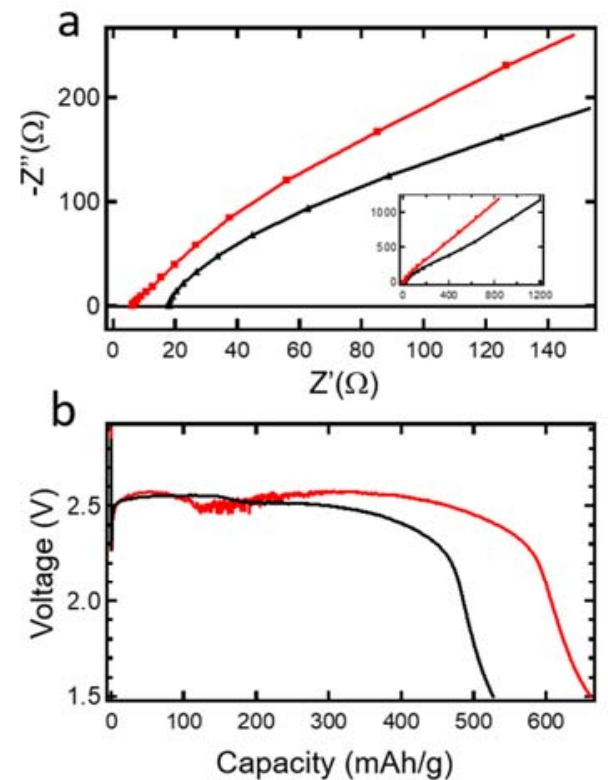

Figure 3. a. High-frequency region of the impedance spectra of $\mathrm{Li} / \mathrm{CF} x$ batteries made with GF (black) and the GF-polyaniline composite materials (red), respectively, in the cathode. Inset: fullrange spectra measured between $200 \mathrm{kHz}$ and $0.1 \mathrm{~Hz}$. b. Discharge curves of the Li/CFx batteries made with GF (black) and the GFpolyaniline composite materials (red), respectively. The discharge rate is $C / 50$. The measurements were stopped when the discharge voltage decreased to $1.5 \mathrm{~V}$ from the initial $2.5 \mathrm{~V}$, with a specific capacity of $526 \mathrm{mAh} \mathrm{g}^{-1}$ (based on mass of GF) and $659 \mathrm{mAh} \mathrm{g}^{-1}$ (based on mass of the GF-polyaniline composite used).

The facile reduction of GF by the weak, non-nucleophilic reductants raises interesting questions regarding the electronaccepting states and the defluorination mechanism. Because of the negligible nucleophilicity of the weak reductants studied, in order to effect $\mathrm{C}-\mathrm{F}$ bond cleavage, clearly the electron-accepting states should have significant orbital overlap with the antibonding $\left(\sigma^{*}\right)$ orbital of the $\mathrm{C}-\mathrm{F}$ bond. ${ }^{19}$ Fully saturated perfluoroalkanes have electron affinities less than $1 \mathrm{eV},{ }^{8}$ too low to account for the observed reduction and suggesting involvement of other structural features that are generally ignored in GF. In particular, residual $\pi$ bonds may exist in GF due to incomplete fluorination in production. This is indicated by XPS in the samples we studied (ESI, Figure S4), which shows peaks at the same C1s binding energy as partly reduced GF. In addition, gray color of GF suggests existence of extended conjugated $\mathrm{C}=\mathrm{C}$ bonds. Studies on small molecules have shown perfluoroalkyl-substituted olefins could have significantly greater electron affinity than perfluoroalkanes or olefins. For example, 1,1,2,2-tetra(trifluoromethyl) ethylene (TTFME) has a reduction potential of $-0.61 \mathrm{~V}$ vs. SCE ( $-0.37 \mathrm{~V}$ vs. SHE, or $4.1 \mathrm{~V}$ below vacuum) in acetonitrile. ${ }^{20}$

To evaluate the feasibility that the residual $\pi$-bonds in GF may be responsible for the electron transfer, we calculated the electron affinity of graphene fluoride containing an ethylene moiety. This was done with nanometer-sized graphene fluoride fragments of various sizes (ESI, e.g., Figure S6) until the calculated electron affinity remains invariant with increasing size. Our calculations yielded an electron affinity of $3.8 \mathrm{eV}$, comparable to the experimental value of TTFME within the uncertainty of the calculations. ${ }^{21}$ The large electron affinity is primarily due to the effective overlap between the $\pi^{*}$ orbital and the $\sigma^{*}$ orbitals of the $\beta$-CF bonds, a phenomenon known as negative anionic hyperconjugation in small-molecule fluorohydrocarbons. ${ }^{22}$ As a result, the greater electronegativity of the fluorine atoms deceases the energy of the $\pi^{*}$ orbital and stabilizes the carbanion. Our estimate of the thermodynamic driving force for the electron transfer from the reductants to GF shows it is possible though likely an uphill process (ESI). The subsequent elimination of $F^{-}$because of the partial occupation of the $\beta$-CF $\sigma^{*}$ orbitals after reduction of $\mathrm{GF}^{22}$ together with the large excess of the reductants present, shifts the equilibrium in the direction favoring the reductive defluorination, consistent with our observations. The reduction and defluorination may proceed repetitively, similar to a reaction sequence proposed for defluorination of perfluorodecalin to yield octafluoronapthalene. ${ }^{23}$ Our calculations show such sequences are energetically feasible (ESI). Defluorination produces either radical species or conjugated alkenes with increasing conjugation length, which have increased electron affinities and make further reduction more favorable. In addition, extended conjugated $\mathrm{C}=\mathrm{C}$ bonds that likely preexist in $\mathrm{GF}$ as indicated by its gray color, can also serve as electron-accepting sites for the reduction. However, we note that due to the complex long-range structures of GF and various bonding conformations possible, our current work only suggests the possible roles of the unsaturated carbon species in the reductive defluorination. A detailed reaction mechanism warrants further investigation.

In summary, we show unexpected reductive defluorination of graphite monofluoride (GF) by weak, non-nucleophilic reductants, indicating the presence of low-lying electron-accepting sites. Further, we could take advantage of the reduction properties of GF to oxidize aniline, a weak reductant, to synthesize GF-polyaniline composites. The use of the composites as cathode materials led to significantly improved discharge capacity of primary lithium batteries. Based on an analysis of the redox energetics, we postulate that the reduction of GF is mediated by residual $\pi$-bonds in GF. The hyperconjugation between the $\pi^{*}$ orbital and the $\sigma^{*}$ orbitals of the $\beta$-CF bonds enhances the electron accepting properties of the materials.

This work is supported by Indiana University and the National Science Foundation (NSF grant CHE-1665427). The XPS measurements were done at the Nanoscale Characterization Facility of Indiana University (NSF grant DMR MRI-1126394).

\section{Conflicts of interest}

The authors have no conflicts to declare.

\section{Notes and references}

1. (a) Dreyer, D. R.; Park, S.; Bielawski, C. W.; Ruoff, R. S., Chem. Soc. Rev. 2010, 39, 228-240; (b) Karlický, F.; Datta, K. K. R.; Otyepka, M.; Zbořil, R., ACS Nano 2013, 7, 6434-6464; (c) Pumera, M.; Wong, C. H. A., Chem. Soc. Rev. 2013, 42, 5987-5995.

2. Ruff, O.; Bretschneider, O., Z. Anorg. Allg. Chem. 1934, 217, 118. 
3. (a) Whittingham, M. S., J. Electrochem. Soc. 1975, 122, 526-527;

(b) Touhara, H.; Fujimoto, H.; Watanabe, N.; Tressaud, A., Solid

State lonics 1984, 14, 163-170.

4. Zhang, Q.; Takeuchi, K. J.; Takeuchi, E. S.; Marschilok, A. C., Phys. Chem. Chem. Phys. 2015, 17, 22504-22518.

5. (a) Robinson, J. T.; Burgess, J. S.; Junkermeier, C. E.; Badescu, S. C.; Reinecke, T. L.; Perkins, F. K.; Zalalutdniov, M. K.; Baldwin, J. W.; Culbertson, J. C.; Sheehan, P. E.; Snow, E. S., Nano Lett. 2010, 10, 3001-3005; (b) Nair, R. R.; Ren, W.; Jalil, R.; Riaz, I.; Kravets, V. G.; Britnell, L.; Blake, P.; Schedin, F.; Mayorov, A. S.; Yuan, S.; Katsnelson, M. I.; Cheng, H.-M.; Strupinski, W.; Bulusheva, L. G.; Okotrub, A. V.; Grigorieva, I. V.; Grigorenko, A. N.; Novoselov, K. S.; Geim, A., Small 2010, 6, 2877-2884; (c) Zbořil, R.; Karlický, F.; Bourlinos, A. B.; Steriotis, T. A.; Stubos, A. K.; Georgakilas, V.; Šafářová, K.; Jančík, D.; Trapalis, C.; Otyepka, M., Small 2010, 6, 2885-2891.

6. Lee, W.-K.; Haydell, M.; Robinson, J. T.; Laracuente, A. R.; Cimpoiasu, E.; King, W. P.; Sheehan, P. E., ACS Nano 2013, 7, 62196224.

7. (a) Rüdorff, W.; Rüdorff, G., Z. Anorg. Chem. 1947, 253, 281296; (b) Ebert, L. B.; Brauman, J. I.; Huggins, R. A., J. Am. Chem. Soc. 1974, 96, 7841-7842; (c) Kita, Y.; Watanabe, N.; Fujii, Y., J. Am. Chem. Soc. 1979, 101, 3832-3841; (d) Touhara, H.; Kadono, K.; Fujii, Y.; Watanabe, N., Z. Anorg. Allg. Chem. 1987, 544, 7-20; (e) Charlier, J.-C.; Gonze, X.; Michenaud, J.-P., Phys. Rev. B 1993, 47, 1616216168; (f) Han, S. S.; Yu, T. H.; Merinov, B. V.; van Duin, A. C. T.; Yazami, R.; Goddard, W. A. I., Chem. Mater. 2010, 22, 2142-2154.

8. Leenaerts, O.; Peelaers, H.; Hernández-Nieves, A. D.; Partoens, B. P., F. M, Phys. Rev. B 2010, 82, 195436.

9. (a) Zbořil, R.; Karlický, F.; Bourlinos, A. B.; Steriotis, T. A.; Stubos, A. K.; Georgakilas, V.; Šafářová, K.; Jančík, D.; Trapalis, C.; Otyepka, M., Small 2010, 6, 2885-2891; (b) Whitener Jr., K. E.; Stine, R.; Robinson, J. T.; Sheehan, P. E., J. Phys. Chem. C 2015, 119, 1050710512.

10. Tuttle, T. R., J. Chem. Phys. 1959, 30, 331-331.

11. Jonsson, M.; Houmam, A.; Jocys, G.; Wayner, D. D. M., J. Chem. Soc., Perkin Trans. 2 1999, 425-429.

12. Trasatti, S., Pure \& Appl. Chem. 1986, 58 (7), 955-966.

13. Zanello, P.; Cinquantini, A.; Mangani, S.; Opromolla, G.; Pardi, L.; Janiak, C.; Rausch, M. D., J. Organomet. Chem. 1994, 471, 171-177. 14. (a) Ashton, P. R.; Balzani, V.; Becher, J.; Credi, A.; Fyfe, M. C. T.; Mattersteig, G.; Menzer, S.; Nielsen, M. B.; Raymo, F. M.; Stoddart, J. F.; Venturi, M.; Williams, D. J., J. Am. Chem. Soc. 1999, 121, 39513957; (b) Wudl, F.; Smith, G. M.; Hufnagel, E. J., Chem. Comm. 1970, 1453-1454.

15. Pedersen, A.; Skagestad, V.; Tilset, M., Acta Chem. Scand. 1995, 49, 632-635.

16. Beams, R.; Cancado, L. G.; Novotny, L., J. Phys.: Conden. Mat. 2015, 27, 083002.

17. Sun, Y.; MacDiarmid, A. G.; Epstein, A. J., J. Chem. Soc., Chem. Commun. 1990, 529-531.

18. Konaka, R.; Kururna, K.; Terabe, S., J. Am. Chem. Soc. 1968, 90, 1801-1806.

19. (a) Maletin, Y. A.; Cannon, R. D., Theor. \& Exp. Chem. 1998, 34, 57-68; (b) Amii, H.; Uneyama, K., Chem. Rev. 2009, 109, 2119-2183. 20. Corvaja, C.; Farnia, G.; Formenton, G.; Navarrini, W.; Sandona, G.; Tortelli, V., J. Phys. Chem. 1994, 98, 2307-2313.

21. Noffke, B. W.; Li, Q.; Raghavachari, K.; Li, L.-S., J. Am. Chem. Soc. 2016, 138, 13923-13929.

22. Schleyer, P. v. R.; Kos, A. J., Tetrahedron 1983, 39, 1141-1150.

23. Sandford, G., Tetrahedron 2003, 59, 437-454. 